\title{
Strain: Una ventana a la mecánica ventricular.
}

\author{
Manuel Pinto.
}

\section{Aspectos Generales}

Una de las maravillas de la estructura y función cardíacas, es el acortamiento sistólico de la fibra miocárdica de alrededor de $15 \%$, que se traduce en un engrosamiento radial de la pared de un $40 \%$,y finalmente se expresa en un cambio de volumen de la cavidad ventricular de un $60 \%$ en un corazón normal ${ }^{1}$.

Para llegar a esta conclusión han pasado cientos de años de estudio de la anatomía cardíaca, desde Richard Lower en 1670 quien describió la disposición helicoidal de las fibras miocárdicas, hasta Francisco Torrent-Guasp quien, mediante disección roma del corazón, demuestra la existencia de una banda miocárdica única ${ }^{2}$, en la que confluyen las fibras miocárdicas formando una doble espiral. En el ventrículo izquierdo describe fibras descendentes subendocárdicas y ascendentes subepicárdicas, ambas en un ángulo de aproximadamente 60 grados y perpendicular entre ellas. Entre ambas capas existe una tercera de fibras circunferenciales que por ser perpendiculares al ultrasonido en la visión de cuatro cavidades son visibles en el septum interventricular como una ecodensidad lineal mayor a nivel de su parte media.

En sístole las fibras descendentes y ascendentes se mueven en dirección contraria. Debido a la contracción de las descendentes (y simultáneo estiramiento de las ascendentes), inicialmente en la sístole existe acercamiento de la base del ventrículo hacia el ápex, que realiza un movi- miento de rotación en sentido anti horario (si se observa al corazón desde la punta) simultáneamente con la rotación horaria de la base ("estruje de la cavidad"). La contracción de las fibras subepicárdicas ascendentes devuelven la base a su posición de reposo, que debido a su mayor radio de movimiento y torque determina la torsión global anti horaria del ventrículo izquierdo y su posterior detorsión, que contribuye a la succión activa de la sangre auricular apenas se abre la válvula mitral ${ }^{3,4}$. De ello resulta que la relajación ventricular comienza en sístole con la contracción de las fibras ascendentes.

\section{Strain}

(Ver en este número:

Rev Chil Cardiol 2011; 31: 89-94 y Rev Chil Cardiol 2011; 31: 95-102)

Desde sus inicios la ecocardiografía se ha convertido en una importante herramienta de valoración de la función cardíaca. La ecocardiografía, tiene como fin la valoración cualitativa y cuantitativa de la morfología y la función que pueden alterarse por la enfermedad. La aparición de nuevas modalidades de visualización ecocardiográficas como el Doppler Tisular (DT) y Strain (o deformación) que permiten el estudio de la mecánica miocárdica casi a nivel de las fibras miocárdicas nos ofrece la posibilidad de detectar mejor los mecanismos envueltos, el pronóstico y potencialmente influenciar el tratamiento.
Correspondencia:

Dr. Manuel Pinto Soazo.

Departamento de Cardiología.

Clínica Alemana de Santiago

mpinto@alemana.cl 
La introducción del Doppler Tisular ${ }^{5}$ ha permitido obtener información sobre la velocidad y desplazamiento del miocardio a lo largo del ciclo cardíaco, y se ha convertido en una importante ayuda en el estudio de la función diastólica y en la valoración de la sincronía. Tiene algunas limitaciones como la dependencia del ángulo de incidencia del ultrasonido sobre la región en estudio, de las variaciones respiratorias, del efecto de tracción que ejercen los segmentos normales vecinos sobre los adyacentes anormales, y la influencia de la movilidad general del corazón (translación, torsión y rotación). El DT estudia velocidades del miocardio que se acercan o alejan del transductor fundamentalmente longitudinales, que por derivación o integración de éstas nos dan información que permite estimar el desplazamiento segmentario (Tissue Tracking) en milímetros, la sincronía tisular en milisegundos (TSI), el Strain o deformación segmentaria, en porcentaje, y el Strain Rate o velocidad de la deformación (en $\mathrm{cm} / \mathrm{s}$ ). Estas últimas mediciones evitan el efecto de tracción de los segmentos normales sobre anormales. Sin embargo, por su origen Doppler están limitadas a efectuarse sólo en la dirección del ultrasonido, y no nos da información radial, circunferencial ni de la rotación ventricular. Estas mediciones informan lo que sucede en puntos ubicados en la región de interés elegida, que habitualmente están en los segmentos basales, medios y apicales de cada pared ventricular, y que permiten su comparación simultánea.

Un paso importante ha sido la incorporación del Strain no Doppler, también denominado Speckle Tracking (o Strain 2D) basado en la imagen bidimensional habitual y que utiliza las irregularidades acústicas o gránulos blancos y negros de la imagen denominados "speckles" que se observan en el miocardio como resultado de la interacción entre el tejido y el ultrasonido y que cambian de posición en el tiempo. Estos marcadores acústicos son captados y seguidos (tracking) en su desplazamiento en el espacio lo que permite medir la velocidad del tejido, strain y strain rate y nos informa sobre movimiento y deformación tanto global como segmentaria. Durante la contracción, el miocardio se acorta longitudinalmente pero debe engrosarse en el sentido transversal (siguiendo la ley de la conservación de la masa), lo que produce acercamiento o deformación relativa de los speckles en sístole que es lo esperable y normal, y en cuyo caso se establece un valor negativo del strain o porcentaje de proximidad relativa que alcanzan. De esta forma se logra el strain en dirección longitudinal (como en Strain DT) y el radial o transversal, si la imagen se obtiene desde el ápex. Si la imagen la adquirimos desde paraesternal en el eje corto se logra el strain radial y circunferencial (tangencial). Con la adquisi- ción del eje corto basal y apical del ventrículo izquierdo se obtiene además la rotación apical y basal y su diferencia que es la torsión, lo que completa el estudio de la mecánica ventricular. Además de los strain longitudinal, radial y circunferencial, existen otros tres, los strain de deslizamiento ("shear strain"), que son los circunferencial-longitudinal, circunferencial-radial y longitudinal-radial que por su efecto amplificador explican el engrosamiento radial de la pared de $40 \%$ y el $60 \%$ de fracción de eyección. El grado de deslizamiento aumenta hacia el subendocardio lo que determina su mayor engrosamiento. El deslizamiento miocárdico en el plano circunferencial-longitudinal durante la eyección resulta en la deformación torsional de ventrículo izquierdo y es de máxima importancia en la mantención de la función sistólica y diastólica, así como de la sincronía ${ }^{6}$.

El Speckle Tracking (Strain 2D), tiene dos variaciones una el Strain bidimensional y la denominada AFI (automatic functional imaging), y es una técnica que ha sido validada para el estudio de la deformación miocárdica mediante sonomicrometría ${ }^{7}$ y resonancia magnética ${ }^{8}$. Se efectúa "off-line" sobre imágenes bidimensionales previamente adquiridas con el "frame rate" adecuado. Para evitar subestimación de mediciones longitudinales y transversales de ventrículo izquierdo debe contener el "verdadero ápex" en las visiones apicales o un eje corto circular desde paraesternal para un estudio correcto de la deformación radial, circunferencial, y de la rotación. En el modo strain bidimensional (desde el ápex o eje corto) se dibuja manualmente el endocardio ventricular o bien se ubican puntos en las bases y el ápex en las tres visiones apicales en el caso del AFI. El equipo procesa esta información y origina un área de interés que abarca el grosor de la pared. En general el strain se mide en el máximo valor obtenido (negativo si el segmento se acorta y positivo si se elonga) lo que habitualmente ocurre en coincidencia con el cierre aórtico. Para el estudio segmentario del ventrículo izquierdo las paredes se dividen automáticamente en segmentos basales, medios y apicales y el equipo da el valor de strain para cada uno de esos segmentos. Es posible desplegar los valores en un mapa polar que da una visión global del strain segmentario y su distribución en el ventrículo (por ejemplo en la distribución anatómica de la arteria descendente anterior), del strain longitudinal máximo en 4, 3 y 2 cavidades apical y el promedio global máximo, que es el valor utilizado habitualmente en los estudios sobre aplicación clínica.

Tanto el Strain DT como el Speckle tracking (Strain 2D) se aplican no sólo al ventrículo izquierdo, sino que también al ventrículo derecho y la aurícula izquierda. Sin 
embargo el Strain DT tiene limitaciones en el estudio y resultados del ápex por lo que habitualmente no se utiliza en este segmento, y comparte con el Speckle tracking la posibilidad de ser influenciado por el complejo movimiento tridimensional de las cavidades cardíacas. En la aurícula izquierda pueden haber limitaciones en las mediciones debidas al escaso grosor de sus paredes especialmente del septum interauricular, en la entrada de las venas pulmonares y la orejuela izquierda. La salida de la aorta dificulta las mediciones de la aurícula izquierda en el equivalente a tres cavidades.

Recientemente se ha agregado a los anteriores otro tipo de strain, el Strain Tridimensional (3D) también basado en seguimiento de speckles, que tiene la capacidad de analizar los tres componentes del desplazamiento local, y evitar el efecto de la translación cardíaca pudiendo seguir el movimiento de los speckles en cualquier dirección ${ }^{9}$. Dado lo anterior las mediciones de volúmenes ventriculares con Strain 3D ${ }^{10}$ están en cercana concordancia con resonancia magnética y mejores que con Strain 2D. Para el estudio Strain 3D se adquieren imágenes de "volumen completo" desde el apex en la forma de una pirámide en la que está contenido el ventrículo o todo el corazón. Con la tecnología actual la adquisición puede tomar desde un ciclo hasta siete ciclos (sub volúmenes o porciones del volumen completo), que son fusionados en uno sólo por el instrumento. El proceso de las imágenes en estos equipos es también semiautomático, y permite alinearlas y obtener el verdadero apex con el toque de un botón. Colocando un punto en la base del ventrículo y otro en el apex tanto en sístole como en diástole el instrumento detecta los bordes endocárdicos y establece una región de interés que abarca todo el grosor de las paredes del ventrículo izquierdo. El seguimiento de los speckles en el ciclo cardíaco permite obtener información del movimiento y deformación tanto segmentaria como global y sus valores se despliegan de igual forma que para Strain 2D. Se obtienen el strain longitudinal, radial y circunferencial en forma automática, en el mismo ciclo, al que recientemente se ha agregado el strain de área, que une información de strain longitudinal y circunferencial para describir mejor el comportamiento segmentario del ventrículo.

El principal problema de la técnica es la dependencia en la calidad de la imagen. Como en el caso del Strain 2D es necesario verificar la calidad de la información que da el instrumento y efectuar los ajustes manuales necesarios si no se está conforme. Otra limitación actual es el frame rate que es menor que el del Strain 2D y del Strain DT lo que limita el estudio de eventos que suceden muy rápido. Además, a pesar de ser una técnica validada con sonomi- crometría en animales ${ }^{11}$ en este momento no hay un método no invasivo de comparación estándar que permita su validación en el ser humano.

\section{Uso clínico del Strain}

La mecánica longitudinal del ventrículo izquierdo depende predominantemente del subendocardio, que es el más vulnerable y sensible a la presencia de enfermedad miocárdica. La función de las capas media y epicárdica puede permanecer relativamente normal inicialmente por lo que el strain circunferencial y la torsión pueden mantenerse iguales o incluso tener un comportamiento exagerado compensatorio para mantener la función sistólica. La disminución de la distensibilidad ventricular produce alteración de la relajación longitudinal y progresivo retardo de la detorsión que altera la función diastólica y eleva las presiones de llenado en una fase en que la fracción de eyección se mantiene aún normal. Por otro lado, el compromiso transmural agudo o progresivo lleva a una disfunción subepicárdica y de la capa media con deterioro de la mecánica circunferencial, de la torsión, y de la fracción de eyección.

Por lo tanto, la detección de alteración de la mecánica longitudinal exclusive puede ser suficiente para identificar precozmente la presencia de enfermedad miocárdica. El análisis del strain radial, circunferencial y de la mecánica torsional nos permite caracterizar el compromiso transmural lo que arroja evidencias sobre el mecanismo de la disfunción. Por ejemplo, en la pericarditis constrictiva que produce tracción del subepicardio se compromete la mecánica circunferencial y la torsión. En el caso de la radioterapia el efecto ocurre no sólo en el pericardio sino que alcanza al subendocardio por lo que ocurre atenuación de la función circunferencial y longitudinal.

En la enfermedad coronaria es el subendocardio el área más vulnerable a la isquemia por lo que es la disminución del strain longitudinal el que manifiesta este hecho aun cuando el engrosamiento de la pared parezca visualmente normal ${ }^{12}$. En el infarto del miocardio de pequeña extensión, tanto el strain DT como el 2D muestran disminución longitudinal y radial segmentaria de su valor, sin compromiso circunferencial ni de la rotación. Si es de mayor extensión y transmural disminuye también el strain circunferencial. Además, con esta técnica es posible detectar la viabilidad miocárdica en territorios infartados. Dado que en el desarrollo de isquemia se aprecia reducción del strain longitudinal máximo, del circunferencial y también del radial, tiene también un papel en la cuantificación ecocardiográfica en estrés con dobutamina ${ }^{3}$. 
Una frecuente aplicación es la hipertrofia ventricular izquierda en la que se ha usado para detectar cambios subclínicos del miocardio y para diferenciar sus diferentes variedades.

La hipertrofia fisiológica del deportista se caracteriza en general por aumento significativo de todos los strain.

En la cardiopatía hipertensiva el strain 2D longitudinal esta disminuido, mientras el radial y circunferencial están preservados. La torsión no cambia pero la detorsión puede estar disminuida y retardada ${ }^{14}$.

La miocardiopatía hipertrófica, caracterizada por el desarreglo miofibrilar, muestra disminución del strain DT y 2D longitudinal. Dependiendo de la ubicación y cuantía de la fibrosis puede haber compensación variable del strain circunferencial a la reducción longitudinal del strain. La detorsión apical en estos casos está significativamente disminuida

La miocardiopatía dilatada se caracteriza por reducción de todos los tipos de strain.

En la Insuficiencia Cardíaca diastólica se observa disminución del strain longitudinal global, con variaciones en el strain radial y circunferencial, y con la progresión del proceso a disfunción sistólica se produce reducción tanto de la torsión máxima de ventrículo izquierdo como de la detorsión que se correlacionan con el grado de disfunción sistólica y diastólica. Esto apoya a la hipótesis que señala que la insuficiencia cardiaca es un mismo proceso que se desarrolla en el tiempo ${ }^{15}$. Por otro lado, un strain longitudinal global muy deprimido en pacientes con disfunción ventricular izquierda permitiría una mejor estratificación de riesgo que la fracción de eyección ${ }^{16}$.

En la terapia de resincronización (TRV), si bien hasta el momento no existe un índice ecocardiográfico recomendado para indicarla, existe interés en el uso del strain 2D en el estudio de la sincronía ya que ha permitido valorar los componentes del movimiento radial, circunferencial y longitudinal para predecir la respuesta a la TRV, sien- do la medición radial y longitudinal en conjunto las más exactas ${ }^{17}$. Los respondedores en general muestran una significativa mejoría de los strain con la remodelación reversa del ventrículo izquierdo. Sin embargo, aún no hay consenso sobre cómo estos índices de mecánica ventricular deben aplicarse en pacientes que requieren TRV.

Las características del Strain y del Strain rate permiten detectar el compromiso subclínico de la función ventricular en diversas patologías como en la diabetes en la que es posible detectar reducciones del strain longitudinal y exageración de la torsión ventricular como respuesta compensadora antes que se haga evidente cualquier otro signo de compromiso ecocardiográfico .

En la cardiotoxicidad por quimioterápicos como trastuzumab y antraciclinas ${ }^{18}$ se puede detectar daño subclínico en pacientes con fracción de eyección normal y podría ayudar a decidir la suspensión eventual del tratamiento

\section{Conclusión}

En suma, Strain es una técnica recientemente incorporada al estudio de la función ventricular y su mecánica, que tiene particularidades que la hacen atractiva en muchos ámbitos de la patología cardíaca, pero que aún requiere de mayor experiencia en su aplicación clínica. Existe evidencia actual, sin embargo que indica que el estudio de la deformación con Strain DT o Speckle tracking da información adicional que no se obtiene con otras técnicas. Para el ecocardiografista representa un reto pues su uso implica incorporar conceptos nuevos, integrar nuevas técnicas a su esquema de trabajo y en este momento dedicar tiempo extra al estudio ecocardiográfico habitual. Este reto debe ser asumido por los ecocardiografistas, quienes además debemos integrar los datos de los diferentes tipos de strain obtenidos, en el informe que se entrega al paciente y a su médico, para que este pueda incrementar la información no sólo diagnostica, sino pronóstica y probablemente influir en la conducta terapéutica. 


\section{Referencias:}

1. COVELL JW. Tissue structure and ventricular wall mechanics. Circulation. 2008; 118:699

2. BALLESTER M, FERREIRA A, CARRERAS F. The Myocardial Band. Heart Failure Clin. 2008;4:261-272

3. TORRENT-GUASP F. La mecánica agonista-antagonista de los segmentos descendente y ascendente de la banda miocárdica ventricular. Rev Esp Cardiol 2001; 54: 1091-102

4. SENGUPTA PP, TAJIK AJ, CHANDRASEKARAN K, KHANDHERIA BK. Twist mechanics of the left ventricle: principles and application. JACC Cardiovasc Imaging 2008; 1: 366-76

5. SUTHERLAND GR, HATLE L, CLAUS P, D’HOOGE J, BIJNENS BH. Doppler Myocardial Imaging. A textbook. Hasselt, Belgium: BSWK; 2006.

6. GEYER H, CARACCIOLO G, ABE H, WILANSKY S, CARERJ S, GENTILE F, et al. Assessment of Myocardial Mechanics Using Speckle Tracking Echocardiography: Fundamentals and Clinical Applications. J Am Soc Echocardiogr 2010;23:351-69

7. PIRAT B, KHOURY DS, HARTLEY CJ, TILLER L, RAO L, SCHULZ DG, et al. A novel feature-tracking echocardiographic method for the quantitation of regional myocardial function: validation in an animal model of ischemia-reperfusion. J Am Coll Cardiol 2008;51:651-9.

8. AMUNDSEN BH, HELLE-VALLE T, EDVARDSEN T, TORP $\mathrm{H}, \mathrm{CROSBY}$ J, LYSEGGEN E, et al. Noninvasive myocardial strain measurement by speckle tracking echocardiography: validation against sonomicrometry and tagged magnetic resonance imaging. J Am Coll Cardiol 2006;47:789-93.

9. MAFFESSANTI F, NESSER HJ, WEINERT L, STERINGER-MASCHERBAUER R, NIEL J, GORISSEN W, et al. Quantitative evaluation of regional left ventricular function using three-dimensional speckle tracking echocardiography in patients with and without heart disease. Am J Cardiol 2009; 104:1755-62.

10. NESSER HJ, MOR-AVI V, GORISSEN W, WEINERT L, STERINGER-MASCHERBAUER R, NIEL J, et al. Quantification of left ventricular volumes using three dimensional echocar- diographic speckle tracking: comparison with MRI. Eur Heart J 2009;30:1565-73.

11. SEO Y, ISHIZU T, ENOMOTO Y, SUGIMORI H, YAMAMOTO M, MACHINO T, et al. Validation of 3-dimensional speckle tracking imaging to quantify regional myocardial deformation. Circ Cardiovasc. Imaging 2009;2:451-9. .

12. CHOI JO, CHO SW, SONG YB, CHO SJ, SONG BG, LEE $\mathrm{SC}$, et al. Longitudinal2D strain at rest predicts the presence of left main and three vessel coronaryartery disease patients without regional wall motion abnormality. Eur J Echocardiogr 2009;10:695-701.

13. HANEKOM L, CHO GY, LEANO R, JEFFRIESS L, MARWICK TH. Comparison oftwo-dimensional speckle and tissue Doppler strain measurement during dobutamine stress echocardiography: an angiographic correlation. Eur Heart J 2007; 28:1765-72.

14. TAKEUCHI M, BORDEN WB, NAKAI H, NISHIKAGE T, KOKUMAI M, NAGAKURA T, et al. Reduced and delayed untwisting of the left ventriclein patients with hypertension and left ventricular hypertrophy: a studyusing two-dimensional speckle tracking imaging. Eur Heart J 2007; 28:2756-62.

15. BRUTSAERT DL. Cardiac dysfunction in heart failure: the cardiologist's loveaffair with time. Prog Cardiovasc Dis 2006;49:157-81.

16. MIGNOT A, DONAL E, ZAROUI A, REANT P, SALEM A, HAMON C, et al. A Global Longitudinal Strain as a Major Predictor of Cardiac Events in Patients with Depressed LeftVentricular Function: A Multicenter Study. J Am Soc Echocardiography 2010;23:1019-24.

17. GORCSAN J 3RD, TANABE M, BLEEKER GB, SUFFOLETTO MS, THOMAS NC, SABA S, et al. Combined longitudinal and radial dyssynchrony predicts ventricular response after resynchronization therapy. J Am Coll Cardiol 2007; 50:1476-83

18. JASSAL DS, HAN SY, HANS C, SHARMA A, FANG T, AHMADIE R, et al, Utility of Tissue Doppler and Strain Rate Imaging in the Early Detection of Trastuzumab and Anthracycline Mediated Cardiomyopathy. J Am SocEchocardiogr 2009;22:418-424. 